\title{
THE GEOMETRIC GENUS OF A DESINGULARIZED FIBER PRODUCT OF ELLIPTIC SURFACES
}

\begin{abstract}
CHAD SCHOEN
(Communicated by Lev Borisov)

Abstract. A formula for the geometric genus is given under the assumption of tame ramification.
\end{abstract}

\section{INTRODUCTION}

Desingularized fiber products of elliptic surfaces with section have proven to be a relatively tractable class of threefolds which have found applications in areas as diverse as algebraic cycles [17, modularity of Galois representations [12, Chapter 2], phenomena peculiar to positive characteristics [16], and superstring theory [5]. For further progress, a simple and general formula for the geometric genus should be very helpful. The purpose of this note is to supply such a formula in the tamely ramified case.

To describe precisely the threefolds of interest we begin with an algebraically closed field, $k$, and a smooth, projective, connected curve, $X / k$, whose function field will be denoted by $K$. Let $E$ and $E^{\prime}$ be elliptic curves over $K$ whose $J$-invariants do not lie in $k$. In addition assume that the actions of the absolute Galois group, $\operatorname{Gal}\left(K_{\text {sep }} / K\right)$ on the l-torsion subgroups, $E\left(K_{\text {sep }}\right)[l]$ and $E^{\prime}\left(K_{\text {sep }}\right)[l]$, are tamely ramified for some (and hence any) prime $l$ distinct from $\operatorname{char}(k)$, [14, II.7-10], [19, IV.10.2c]. This condition is automatically satisfied whenever $\operatorname{char}(k) \notin\{2,3\}$ [19, IV.10.2b]. Let $\mathbf{K}$ denote the function field of $E \times_{K} E^{\prime}$. It is finitely generated of transcendence degree 3 over $k$. By resolution of singularities for threefolds over $k$ there exists a smooth, projective $k$-variety, $W$, such that $k \subset \mathbf{K}$ and $k \subset k(W)$ are isomorphic field extensions. The geometric genus, $p_{g}:=\operatorname{dim}_{k}\left(H^{0}\left(W, \Omega_{W / k}^{3}\right)\right)$, is independent of the choice of the smooth, projective model of $\mathbf{K}$ [9, II.8.19]. The main result of this note is a formula for $p_{g}$ in terms of the Kodaira types of the reductions of $E$ and $E^{\prime}$ at closed points $x \in X$ [19, §IV.8].

Notation. $\Delta_{x} \in \mathcal{O}_{X, x}$ (respectively $\Delta_{x}^{\prime}$ ) denotes the discriminant of a minimal Weierstrass model of $E$ (respectively $E^{\prime}$ ) at $x$. Although $\Delta_{x}$ depends on the choice of minimal Weierstrass model, $\operatorname{ord}_{x}\left(\Delta_{x}\right)$ does not:

$o_{x}:= \begin{cases}0, & \text { if } E \text { has semi-stable reduction at } x, \\ \operatorname{ord}_{x}\left(\Delta_{x}\right), & \text { if } E \text { has reduction type II, III, IV, } \mathrm{IV}^{*}, \mathrm{III}^{*}, \text { or } \mathrm{II}^{*} \text { at } x, \\ 6, & \text { if } E \text { has type } \mathrm{I}_{n}^{*}(n \geq 0) \text { reduction at } x .\end{cases}$

Received by the editors January 30, 2011 and, in revised form, July 10, 2011.

2010 Mathematics Subject Classification. Primary 14J30, 14D99, 14E99. 
In effect $o_{x}$ measures that part of the order of vanishing of $\Delta_{x}$ which is attributable to non-semi-stability at $x$. Define $o_{x}^{\prime}$ analogously with $E^{\prime}$ in place of $E$.

$|X|_{0}=$ the set of closed points of $X$.

$g_{X}=$ the genus of $X$.

Definition. $E \times E^{\prime}$ is said to have contributing reduction at $x \in X$ if $o_{x}+o_{x}^{\prime} \geq 12$.

Let $\mathbf{s} \subset X$ denote the subset of points with contributing reduction.

Theorem. $p_{g}=-|\mathbf{s}|+g_{X}-1+\frac{1}{12} \sum_{x \in|X|_{0}}\left(\operatorname{ord}_{x}\left(\Delta_{x}\right)+\operatorname{ord}_{x}\left(\Delta_{x}^{\prime}\right)\right)$.

The theorem makes it possible to immediately read off the geometric genus of $W$ from the reduction types of $E$ and $E^{\prime}$ using the middle row of Table 1 .

TABLE 1

$\begin{array}{ccccccccc} & \mathrm{I}_{n} & \mathrm{II} & \mathrm{III} & \mathrm{IV} & \mathrm{IV}^{*} & \mathrm{III}^{*} & \mathrm{II}^{*} & \mathrm{I}_{n}^{*} \\ \text { bad char. } & - & \{2,3\} & 2 & 3 & 3 & 2 & \{2,3\} & 2 \\ \operatorname{ord}_{x}\left(\Delta_{x}\right) & n & 2 & 3 & 4 & 8 & 9 & 10 & 6+n \\ m_{x} & 1 & 6 & 4 & 3 & 3 & 4 & 6 & 2\end{array}$

The first row lists the bad characteristics for each reduction type. In these characteristics the indicated reduction type forces wild ramification [15, 3.11], which is incompatible with the tame ramification hypothesis. If the characteristic of $k$ is not bad, then the next row gives the order of vanishing of the discriminant [19, $\mathrm{p}$. $365]$ and the final row gives the minimal degree of a field extension of $K$ which is totally ramified at $x$ such that the base change of $E$ acquires semi-stable reduction over $x$. In fact using Tate's algorithm [18, $\S I V .9]$ one may check that $E$ acquires semi-stable reduction over $x$ for every totally ramified field extension of degree $m_{x}$. Except in the cases of the first and last columns in the table, the resulting semi-stable reduction is actually good reduction.

Let $\pi: Y \rightarrow X$ (respectively $\pi^{\prime}: Y^{\prime} \rightarrow X^{\prime}$ ) denote the relatively minimal regular model of $E$ (respectively $E^{\prime}$ ) [11, 9.4.34]. The sheaf $L:=R^{1} \pi_{*} \mathcal{O}_{Y}$ (respectively $L^{\prime}:=R^{1} \pi_{*}^{\prime} \mathcal{O}_{Y^{\prime}}$ ) is invertible (cf. [11, 9.4.29b and 9.4.35]). It is frequently convenient to reformulate the Theorem in terms of the $l$-adic Euler characteristics of $Y$ and $Y^{\prime}$, or in terms of the Euler characteristic of $L \otimes L^{\prime}$.

Corollary. (i) $p_{g}=g_{X}-1+\frac{1}{12}\left(e(Y)+e\left(Y^{\prime}\right)\right)-|\mathbf{s}|$.

(ii) $p_{g}=h^{3}\left(W, \mathcal{O}_{W}\right)=-\chi\left(L \otimes L^{\prime}\right)-|\mathbf{s}|$.

Proof. (i) Under the hypothesis of tame reduction the $l$-adic Euler characteristic, $e(Y)$, is the sum of the $l$-adic Euler characteristics of the fibers 13, V.2.15(c)]. Using the table one verifies readily that $e\left(\pi^{-1}(x)\right)=\operatorname{ord}_{x}\left(\Delta_{x}\right)$. The same holds for $Y^{\prime}$, so (i) is equivalent to the Theorem.

(ii) The first equality is Serre duality. For the second use $1-g_{X}=\chi\left(\mathcal{O}_{X}\right)$ and $\frac{1}{12}\left(e(Y)+e\left(Y^{\prime}\right)\right)=\chi\left(\mathcal{O}_{Y}\right)+\chi\left(\mathcal{O}_{Y^{\prime}}\right)=2 \chi\left(\mathcal{O}_{X}\right)-\chi(L)-\chi\left(L^{\prime}\right)=\chi\left(\mathcal{O}_{X}\right)-\chi\left(L \otimes L^{\prime}\right)$,

where the first equality is Noether's Theorem [9, V.1.6.1], the second follows from the Leray spectral sequences for $\pi$ and $\pi^{\prime}$, and the third is Riemann-Roch on $X$. 
Additional Notation. P denotes any of the Kodaira types II, III, IV.

$\mathrm{P}^{*}$ denotes any of the Kodaira types $\mathrm{II}^{*}, \mathrm{III}^{*}, \mathrm{IV}^{*}, \mathrm{I}_{n}^{*}(n \geq 0)$.

A-B denotes the reduction of $E \times{ }_{K} E^{\prime}$ at $x \in X$ when $E$ has type A reduction and $E^{\prime}$ has type $\mathrm{B}$ reduction.

Remark. Reductions of types A-B and B-A are contributing when A-B is one of the following types: $\mathrm{P}^{*}-\mathrm{P}^{*}, \mathrm{II}^{*}-\mathrm{II}, \mathrm{III}^{*}-\mathrm{III}, \mathrm{IV}^{*}-\mathrm{IV}, \mathrm{II}^{*}-\mathrm{III}, \mathrm{II}^{*}-\mathrm{IV}, \mathrm{III}^{*}-\mathrm{IV}$.

Reductions of types A-B and B-A are not contributing when A-B is one of the following: P-P, II-III* ${ }^{*}$ II-IV*, III-IV* and $\mathrm{I}_{n}-\mathrm{I}_{n^{\prime}}, \mathrm{I}_{n}-\mathrm{P}, \mathrm{I}_{n}-\mathrm{P}^{*}, \mathrm{P}-\mathrm{I}_{n}^{*}$ for $n, n^{\prime} \geq 0$.

\section{Proof of the Theorem}

Keep the notation of the introduction.

Lemma 2.1. There exists a finite Galois extension, $K \subset \widetilde{K}$, such that the base changed curves, $\widetilde{E} / \widetilde{K}$ and $\widetilde{E}^{\prime} / \widetilde{K}$, both have semi-stable reduction and such that the order of the Galois group is not divisible by char $(k)$.

Proof. Let $m_{x}$ (respectively $m_{x}^{\prime}$ ) denote the number associated to the reduction of $E$ (respectively $E^{\prime}$ ) at $x$ in the last row of Table 1 . Set $n_{x}=\operatorname{lcm}\left(m_{x}, m_{x}^{\prime}\right)$ and $n=\prod_{x \in|X|_{0}} n_{x}$. The product is finite and $\operatorname{char}(k) \nmid n$ by Table 1 and the tame ramification hypothesis. Choose $f \in K^{*}$ such that $\operatorname{ord}_{x}(f)=n / n_{x}$ at each $x \in\left|X_{0}\right|$ for which $n_{x}>1$. Such a choice is possible by the weak approximation theorem [14, II.3.4]. Set $\widetilde{K}=K\left(f^{1 / n}\right)$ and write $h: \widetilde{X} \rightarrow X$ for the normalization of $X$ in $\widetilde{K}$. For $x \in|X|_{0}$ the ramification index of $h$ at any point of $h^{-1}(x)$ is divisible by $m_{x}$ and $m_{x}^{\prime}$. It follows that $\widetilde{E}$ and $\widetilde{E}^{\prime}$ have semi-stable reduction.

Let $\tilde{\pi}: \widetilde{Y} \rightarrow \widetilde{X}$ (respectively $\tilde{\pi}^{\prime}: \widetilde{Y}^{\prime} \rightarrow \widetilde{X}$ ) denote the relatively minimal regular model of $\widetilde{E}$ (respectively of $\widetilde{E}^{\prime}$ ). The fiber product,

$$
\widehat{f}: \widehat{W}:=\tilde{Y} \times \widetilde{X} \widetilde{Y}^{\prime} \rightarrow \widetilde{X},
$$

has at worst isolated ordinary double point singularities. Blowing these up gives a smooth, projective $k$-variety, $\widetilde{W}$, with a canonical morphism, $\widetilde{f}: \widetilde{W} \rightarrow \widetilde{X}$. Set $G:=\operatorname{Gal}(\widetilde{K} / K)$ and assume henceforth $\operatorname{char}(k) \nmid|G|$.

Lemma 2.2. The action of $G$ on the right-hand factor in $E \times_{K} \widetilde{K}$ (respectively $E^{\prime} \times_{K} \widetilde{K}, E \times_{K} E^{\prime} \times_{K} \widetilde{K}$ ) gives rise to a biregular action on $\tilde{Y}$ (respectively $\tilde{Y}^{\prime}$, $\widetilde{W})$.

Proof. The action extends to a biregular action on the Néron model, equivariant with respect to the structure morphism, by the universal mapping property [19, IV.5]. By semi-stability the Néron model of $E \times_{K} \widetilde{K}$ is obtained from $\widetilde{Y}$ by removing finitely many points [19, IV.6.1]. The biregular action on the open subset extends to a biregular action on $\tilde{Y}$ [6, 9.2]. This action is $\widetilde{\pi}$-equivariant and there is an analogous $\widetilde{\pi^{\prime}}$-equivariant action on $\tilde{Y}^{\prime}$. Thus there is a biregular action on the fiber product. It takes the ideal sheaf of the reduced singular locus to itself and thus lifts to the blowup of this ideal sheaf, $\widetilde{W}$ [9, p. 163, Definition].

Proposition 2.3. $H^{3}\left(W, \mathcal{O}_{W}\right) \simeq H^{3}\left(\widetilde{W}, \mathcal{O}_{\widetilde{W}}\right)^{G}$. 
Proof. Write $q: \widetilde{W} \rightarrow \bar{W}$ for the quotient of $\widetilde{W}$ by $G$. The sheaf $\mathcal{O}_{\widetilde{W}}$ has a natural structure of a $G$-sheaf in the sense of $[8, \S 5]$. Write $q_{*}^{G}$ for the composition of $q_{*}$ with the $G$-invariant-subsheaf functor. Then $q_{*}^{G}\left(\mathcal{O}_{\widetilde{W}}\right) \simeq \mathcal{O}_{\bar{W}}$. Note that $q_{*}$ is exact since $q$ is finite. Taking $G$-invariants of sheaves of $k$-modules is exact since $\operatorname{char}(k)$ does not divide $|G|$. Thus $R^{i} q_{*}^{G} \mathcal{O}_{\widetilde{W}}=0$ for $i>0$. Now [8, Corollaire, p. 202] gives the first of two isomorphisms,

$$
H^{3}\left(\widetilde{W}, \mathcal{O}_{\widetilde{W}}\right)^{G} \simeq H^{3}\left(\bar{W}, q_{*}^{G}\left(\mathcal{O}_{\widetilde{W}}\right)\right) \simeq H^{3}\left(\bar{W}, \mathcal{O}_{\bar{W}}\right) .
$$

As $H^{3}\left(W, \mathcal{O}_{W}\right) \simeq H^{0}\left(W, \Omega_{W / k}^{3}\right)^{\vee}$ is a birational invariant of smooth projective threefolds, we may assume that $W$ is obtained from $\bar{W}$ by a resolution of singularities, $\sigma: W \rightarrow \bar{W}$. Resolution of singularities is now known for threefolds in all positive characteristics [3. It has been known for some time in characteristics $>5$ [4] and in arbitrary dimension in characteristic zero [10]. Here we deal only with cyclic quotient singularities where the order of the group is not divisible by the characteristic. Quotient singularities of this sort are rational in the sense that $R^{i} \sigma_{*} \mathcal{O}_{W}=0$ for $i>0$ [2, Theorem 2] ([20] if $\operatorname{char}(k)=0$ ). Thus $H^{3}\left(\bar{W}, \mathcal{O}_{\bar{W}}\right) \simeq H^{3}\left(W, \mathcal{O}_{W}\right)$.

Define $\widetilde{L}:=R^{1} \widetilde{\pi}_{*} \mathcal{O}_{\widetilde{Y}}$ and $\widetilde{L}^{\prime}:=R^{1} \widetilde{\pi}_{*}^{\prime} \mathcal{O}_{\widetilde{Y}^{\prime}}$. These are invertible sheaves. Write $h: \widetilde{X} \rightarrow X$ for the normalization of $X$ in $\widetilde{K}$. The sheaf $\widetilde{L} \otimes \widetilde{L}^{\prime}$ is a $G$-sheaf.

Lemma 2.4. $\operatorname{dim}_{k}\left(H^{3}\left(\widetilde{W}, \mathcal{O}_{\widetilde{W}}\right)^{G}\right)=-\chi\left(h_{*}^{G}\left(\widetilde{L} \otimes \widetilde{L}^{\prime}\right)\right)=-\chi\left(\mathcal{O}_{X}\right)-\operatorname{deg}\left(h_{*}^{G}(\widetilde{L} \otimes\right.$ $\left.\widetilde{L}^{\prime}\right)$ ).

Proof. By the Leray spectral sequence,

$$
H^{3}\left(\widetilde{W}, \mathcal{O}_{\widetilde{W}}\right)^{G} \simeq H^{1}\left(\widetilde{X}, R^{2} \widetilde{f}_{*} \mathcal{O}_{\widetilde{W}}\right)^{G} .
$$

Write $p: \widehat{W} \rightarrow \widetilde{Y}$ (respectively $p^{\prime}: \widehat{W} \rightarrow \widetilde{Y}^{\prime}$ ) for the canonical projections. Then

$$
R^{2} \widetilde{f}_{*} \mathcal{O}_{\widetilde{W}} \simeq R^{2} \widehat{f}_{*} \mathcal{O}_{\widehat{W}} \simeq R^{1} \widetilde{\pi}_{*} R^{1} p_{*}\left(\left(p^{\prime}\right)^{*} \mathcal{O}_{\widetilde{Y}^{\prime}}\right) \simeq R^{1} \widetilde{\pi}_{*} \widetilde{\pi}^{*} R^{1} \widetilde{\pi}_{*}^{\prime} \mathcal{O}_{\widetilde{Y}^{\prime}} \simeq \widetilde{L} \otimes \widetilde{L}^{\prime},
$$

where the first isomorphism comes from the Leray spectral sequence for the blowup, $\widetilde{W} \rightarrow \widehat{W}$, the second from the spectral sequence of the composition of left exact functors, $\widehat{f}_{*}=\pi_{*} \circ p_{*}$ and the isomorphism, $\mathcal{O}_{\widehat{W}} \simeq\left(p^{\prime}\right)^{*} \mathcal{O}_{Y^{\prime}}$. The third isomorphism is flat base change [9, III.9.3] and the fourth is the projection formula [9, III. Ex. 8.3]. Thus

$$
H^{3}\left(\widetilde{W}, \mathcal{O}_{\widetilde{W}}\right)^{G} \simeq H^{1}\left(\widetilde{X}, \widetilde{L} \otimes \widetilde{L}^{\prime}\right)^{G} \simeq H^{1}\left(X, h_{*}^{G}\left(\widetilde{L} \otimes \widetilde{L}^{\prime}\right)\right),
$$

where the final isomorphism is proved as in the previous lemma. The composition,

$$
H^{0}\left(X, h_{*}^{G}\left(\widetilde{L} \otimes \widetilde{L}^{\prime}\right)\right) \rightarrow H^{0}\left(X, h_{*}\left(\widetilde{L} \otimes \widetilde{L}^{\prime}\right)\right) \simeq H^{0}\left(\widetilde{X}, \widetilde{L} \otimes \widetilde{L}^{\prime}\right),
$$

is injective. By Grothendieck duality [11, 6.4.3] $\widetilde{L} \simeq \widetilde{\pi}_{*} \omega_{\widetilde{Y} / \widetilde{X}}^{\vee}$ and $\widetilde{L}^{\prime} \simeq \widetilde{\pi}_{*}^{\prime} \omega_{\widetilde{Y}^{\prime} / \tilde{X}^{\prime}}^{\vee}$. Now $\operatorname{deg}(\widetilde{L})=-\left(\frac{1}{12} \sum_{x \in\left|X_{0}\right|} \operatorname{ord}_{x}\left(\Delta_{x}\right)\right)$ [11, 9.4, Exercise 14.a]. As $\operatorname{ord}_{x}\left(\Delta_{x}\right) \geq 0$ always [18, VII.1] and $\operatorname{ord}_{x}\left(\Delta_{x}\right)>0$ at a pole, $x$, of the $J$-invariant, which exists since $J \notin k, \operatorname{deg}(\widetilde{L})<0$. As $\operatorname{deg}\left(\widetilde{L}^{\prime}\right)<0$ as well, $H^{0}\left(X, h_{*}^{G}\left(\widetilde{L} \otimes \widetilde{L}^{\prime}\right)\right)=0$ and the first equality follows. Since $h_{*}^{G}\left(\widetilde{L} \otimes \widetilde{L}^{\prime}\right)$ is a rank-one subsheaf of a locally free sheaf on a smooth curve, it is invertible and the second equality follows from Riemann-Roch. 
The proof of the Theorem thus reduces to computing

$$
\operatorname{deg}\left(h_{*}^{G}\left(\widetilde{L} \otimes \widetilde{L}^{\prime}\right)\right)=\frac{1}{|G|} \operatorname{deg}\left(h^{*} h_{*}^{G}\left(\widetilde{L} \otimes \widetilde{L}^{\prime}\right)\right) .
$$

Here we argue as in [7]. There is an exact sequence of $\mathcal{O}_{\tilde{X}}$-modules in which the first two terms are invertible:

$$
0 \rightarrow h^{*} h_{*}^{G}\left(\widetilde{L} \otimes \widetilde{L}^{\prime}\right) \rightarrow \widetilde{L} \otimes \widetilde{L}^{\prime} \rightarrow \mathcal{R} \rightarrow 0 .
$$

The final sheaf is supported on the ramification locus of $h$. Write $\ell(\mathcal{R})$ for the length of $\mathcal{R}$. Combining this exact sequence with the previous lemma yields

Corollary 2.5. $h^{3}\left(W, \mathcal{O}_{W}\right)=-\chi\left(\mathcal{O}_{X}\right)-\frac{\operatorname{deg}(\widetilde{L})}{|G|}-\frac{\operatorname{deg}\left(\widetilde{L}^{\prime}\right)}{|G|}+\frac{\ell(\mathcal{R})}{|G|}$.

Write $\widetilde{\Delta}_{\widetilde{x}}$ (respectively $\widetilde{\Delta}_{\widetilde{x}}^{\prime}$ ) for the discriminant of a minimal Weierstrass model of $\widetilde{E}$ (respectively $\widetilde{E}^{\prime}$ ) at $\widetilde{x}$. By Grothendieck duality [11, §6.4.3] and [11, 9.4.26c, 9.4.31, 9.4, Exercise 14a]

$$
-\operatorname{deg}(\widetilde{L})=\operatorname{deg}\left(\widetilde{\pi}_{*} \omega_{\widetilde{Y} / \widetilde{X}}\right)=\frac{1}{12} \sum_{\widetilde{x} \in|\widetilde{X}|_{0}} \operatorname{ord}_{\widetilde{x}}\left(\widetilde{\Delta}_{\widetilde{x}}\right) .
$$

Write $G_{\widetilde{x}} \subset G$ for the stabilizer subgroup of $\widetilde{x}$. Note that $\left|G_{\widetilde{x}}\right|$ is independent of the choice of $\widetilde{x}$ in any given fiber, $h^{-1}(x)$. For $x \in|X|_{0}$ define

$$
d_{x}=\frac{1}{12}\left(\operatorname{ord}_{x}\left(\Delta_{x}\right)-\frac{1}{|G|} \sum_{\widetilde{x} \in h^{-1}(x)} \operatorname{ord}_{\widetilde{x}}\left(\widetilde{\Delta}_{\widetilde{x}}\right)\right) .
$$

Lemma 2.6. $d_{x}$ is determined by the reduction type of $E$ at $x$ as follows:

$$
d_{x}= \begin{cases}0, & \mathrm{I}_{n} \text { reduction }, \\ 1 / m_{x}, & \text { type } \mathrm{P} \text { reduction, } \\ 1-\frac{1}{m_{x}}, & \text { type } \mathrm{P}^{*} \text { reduction },\end{cases}
$$

where $m_{x}$ is the number associated to the reduction of $E$ at $x$ in Table 1.

Proof. For $\mathrm{I}_{n}$ reduction,

$$
d_{x}=\frac{1}{12}\left(n-\frac{1}{|G|} \cdot \frac{|G|}{\left|G_{\widetilde{x}}\right|} \cdot\left|G_{\widetilde{x}}\right| n\right)=0 .
$$

For $\mathrm{I}_{n}^{*}$ reduction,

$$
d_{x}=\frac{1}{12}\left(n+6-\frac{1}{|G|} \cdot \frac{|G|}{\left|G_{\widetilde{x}}\right|} \cdot\left|G_{\widetilde{x}}\right| n\right)=1 / 2=1-\frac{1}{m_{x}} .
$$

In the case of potentially good reduction $\operatorname{ord}_{\widetilde{x}}\left(\widetilde{\Delta}_{\widetilde{x}}\right)=0$. Thus one is reduced to reading off of Table 1 that $\operatorname{ord}_{x}\left(\Delta_{x}\right)=12 / m_{x}$ for type $\mathrm{P}$ reduction and $\operatorname{ord}_{x}\left(\Delta_{x}\right)=$ $12\left(1-\frac{1}{m_{x}}\right)$ for reductions of types $\mathrm{II}^{*}, \mathrm{III}^{*}, \mathrm{IV}^{*}$.

Let $d_{x}^{\prime}$ (respectively $m_{x}^{\prime}$ ) be the counterpart of $d_{x}$ (respectively $m_{x}$ ) for $E^{\prime}$. Define $r_{x}=\ell\left(\left.\mathcal{R}\right|_{h^{-1}(x)}\right) /|G|$ and $\epsilon_{x}=d_{x}+d_{x}^{\prime}-r_{x}$. Using $-\chi\left(\mathcal{O}_{X}\right)=g_{X}-1$ the previous corollary may now be rephrased:

Corollary 2.7. $h^{3}\left(W, \mathcal{O}_{W}\right)=g_{X}-1+\sum_{x \in|X|_{0}}\left(\frac{\operatorname{ord}_{x}\left(\Delta_{x}\right)+\operatorname{ord}_{x}\left(\Delta_{x}^{\prime}\right)}{12}-\epsilon_{x}\right)$.

Lemma 2.8. $\epsilon_{x}=1$ if $E \times E^{\prime}$ has contributing reduction at $x$. Otherwise $\epsilon_{x}=0$. 
Proof. It suffices to verify the entries in the fourth column of Table 2. Here the notation $m_{x}$ and $m_{x}^{\prime}$ has been simplified by dropping the subscript ${ }_{x}$.

TABLE 2

$\begin{array}{ccccc}\text { Reduction at } x & d_{x} & d_{x}^{\prime} & r_{x} & \epsilon_{x} \\ \mathrm{I}_{n}-\mathrm{I}_{n^{\prime}} & 0 & 0 & 0 & 0 \\ \mathrm{P}-\mathrm{I}_{n} & \frac{1}{m} & 0 & \frac{1}{m} & 0 \\ \mathrm{P}^{*}-\mathrm{I}_{n} & 1-\frac{1}{m} & 0 & 1-\frac{1}{m} & 0 \\ \mathrm{P}-\mathrm{P} & \frac{1}{m} & \frac{1}{m^{\prime}} & \frac{1}{m}+\frac{1}{m^{\prime}} & 0 \\ \mathrm{P}-\mathrm{P}^{*} m>m^{\prime} & \frac{1}{m} & 1-\frac{1}{m^{\prime}} & 1-\frac{1}{m^{\prime}}+\frac{1}{m} & 0 \\ \mathrm{P}-\mathrm{P}^{*} m \leq m^{\prime} & \frac{1}{m} & 1-\frac{1}{m^{\prime}} & \frac{1}{m}-\frac{1}{m^{\prime}} & 1 \\ \mathrm{P}^{*}-\mathrm{P}^{*} & 1-\frac{1}{m} & 1-\frac{1}{m^{\prime}} & 1-\frac{1}{m}-\frac{1}{m^{\prime}} & 1\end{array}$

Let $G_{\widetilde{x}} \subset G$ denote the stabilizer of some $\widetilde{x} \in h^{-1}(x)$. Then $r_{x}=\ell\left(\mathcal{R}_{\widetilde{x}}\right) /\left|G_{\widetilde{x}}\right|$. The map of local rings, $\mathcal{O}_{X, x} \rightarrow \mathcal{O}_{\widetilde{X}, \widetilde{x}}$, induces a map on completions, which we may identify with the map, $k[[v]] \rightarrow k[[u]], v \mapsto u^{\left|G_{\widetilde{x}}\right|}$. Fix a generator, $\sigma \in G_{\widetilde{x}}$. There is a primitive $\left|G_{\widetilde{x}}\right|$-th root of unity, $\zeta \in k^{*}$ such that $\sigma(u)=\zeta u$. Since $G_{\widetilde{x}}$ acts on $\widetilde{L}_{\widetilde{x}}$ and $\widetilde{L}_{\widetilde{x}}^{\prime}$ one may choose generators $l \in \widetilde{L}_{\widetilde{x}} \otimes_{\mathcal{O}_{\widetilde{X}, \widetilde{x}}} k[[u]]$ and $l^{\prime} \in \widetilde{L}_{\widetilde{x}}^{\prime} \otimes_{\mathcal{O}_{\widetilde{X}, \widetilde{x}}} k[[u]]$ which are eigenvectors for $\sigma$. The eigenvalue corresponding to $l$ is determined by the action of $\sigma$ on $\widetilde{L}_{\widetilde{x}} \otimes k$. Since $\widetilde{L}$ may be canonically identified with the normal sheaf of the identity section of $Y$ (cf. [9, IV.4.10.7]), $\widetilde{L}_{\widetilde{x}} \otimes k$ is canonically the tangent space at the identity in the fiber $\widetilde{\pi}^{-1}(\widetilde{x})$. Define $r$ and $r^{\prime}$ so that $\left|G_{\widetilde{x}}\right|=m r=m^{\prime} r^{\prime}$. Since $(\mathbb{Z} / m)^{*} \subset\{ \pm 1\}$, there are at most two possibilities for the action of $\sigma$ on the tangent space, $T_{\widetilde{x}} \widetilde{Y}$. With respect to an ordered basis, in which the first vector is dual to $u$ and the second is vertical, $\sigma$ acts by $\operatorname{diag}\left(\zeta^{-1}, \zeta^{-r}\right)$ or $\operatorname{diag}\left(\zeta^{-1}, \zeta^{r}\right)$. The corresponding cyclic quotient singularity in the first case is of type $A_{m, 1}$ and in the second case of type $A_{m-1}$. Explicit resolution of these quotient singularities as in [1, V.10] indicates that the first case corresponds to type $\mathrm{P}$ reduction and the second to type $\mathrm{P}^{*}$ reduction. (When $m=2$ there is only one case and the reduction type is $\mathrm{I}_{n}^{*}$.) Thus $\sigma(l)=\zeta^{t r} l$, where $t=0$ (respectively $-1,1$ ) for reductions of type $\mathrm{I}_{n}$ (respectively $\mathrm{P}, \mathrm{P}^{*}$ ). Defining $t^{\prime}$ in the same way gives $\sigma\left(l^{\prime}\right)=\zeta^{t^{\prime} r^{\prime}} l^{\prime}$.

Choose $s \in \mathbb{Z}_{\geq 0}$ minimal such that $l l^{\prime} u^{s} \in \widetilde{L}_{\widetilde{x}} \otimes_{\mathcal{O}_{\widetilde{X}, \widetilde{x}}} \otimes \widetilde{L}_{\widetilde{x}}^{\prime} \otimes_{\mathcal{O}_{\widetilde{X}, \widetilde{x}}} k[[u]]$ is fixed by the action of $G_{\widetilde{x}}$. Then the map induced by the inclusion,

$$
h^{*} h_{*}^{G}\left(\widetilde{L} \otimes \widetilde{L}^{\prime}\right) \rightarrow \widetilde{L} \otimes \widetilde{L}^{\prime},
$$

on the completion of the stalk at $\widetilde{x}$ is naturally identified with the standard inclusion,

$$
l l^{\prime} u^{s} k[[v]] \otimes_{k[[v]]} k[[u]] \simeq l l^{\prime} u^{s} k[[u]] \rightarrow l l^{\prime} k[[u]] .
$$

The cokernel is isomorphic to $k[[u]] /\left(u^{s}\right)$ and $\ell\left(\mathcal{R}_{\widetilde{x}}\right)=s$. Now $s=-\left(r t+r^{\prime} t^{\prime}\right)$ if $r t+r^{\prime} t^{\prime} \leq 0$ and $s=\left|G_{\widetilde{x}}\right|-\left(r t+r^{\prime} t^{\prime}\right)$ otherwise. Set $\mu=\frac{t}{m}+\frac{t^{\prime}}{m^{\prime}}$. Then

$$
r_{x}=\ell\left(\mathcal{R}_{\widetilde{x}}\right) /\left|G_{\widetilde{x}}\right|=s /\left|G_{\widetilde{x}}\right|= \begin{cases}-\mu & \text { if } \mu \leq 0 \\ 1-\mu & \text { otherwise }\end{cases}
$$


as in the fourth column of Table 2. The lemma follows.

The Theorem follows from Corollary 2.7 and Lemma 2.8 .

Lemma 2.9. For all $x$, $\frac{\operatorname{ord}_{x}\left(\Delta_{x}\right)+\operatorname{ord}_{x}\left(\Delta_{x}^{\prime}\right)}{12}-\epsilon_{x} \geq 0$. Equality holds if and only if the reduction at $x$ is of type $I_{0}-I_{0}, I_{0}^{*}-I_{0}^{*}, I I-I I^{*}, I I I-I I I^{*}$, or $I V-I V^{*}$.

Proof. Straightforward case by case checking.

Lemma 2.10. If $p_{g}(W)=0$, then $g_{X}=0$.

Proof. This is because the summation term in Corollary 2.7 is strictly positive. Indeed by Lemma 2.9 it is non-negative, but every elliptic surface with non-constant $J$-invariant must have at least one fiber of type $\mathrm{I}_{n}$ or $\mathrm{I}_{n}^{*}$ with $n>0$, which makes the sum positive.

We hope to say more about desingularized fiber products with $p_{g}=0$ in future work.

\section{QuadratiC TWISTS AND THE ASSOCIATED FAMILY OF KUMMER SURFACES}

Suppose that $\operatorname{char}(k) \neq 2$. A reference for twists by quadratic field extensions is [18, X.2.4]. Define $H:=\left\{ \pm I d_{E \times_{K} E^{\prime}}\right\} \subset A u t_{K}\left(E \times_{K} E^{\prime}\right)$. Let $V$ be a smooth projective $k$-model of the Kummer surface $\left(E \times_{K} E^{\prime}\right) / H$.

Proposition 3.1. (i) $p_{g}(V)=p_{g}(W)$.

(ii) Let $K \subset K^{\prime}$ be a degree two field extension. Simultaneous quadratic twisting of $E$ and $E^{\prime}$ by this field extension does not change $p_{g}$.

Proof. (i) Let $K \subset \widetilde{K}$ be as in Lemma 2.1. Now $-I d_{E}$ acts on the first factor in $E \times_{K} \widetilde{K}=\widetilde{E}$. The involution extends to the Néron model by its universal property [19, IV.5] and then to all of $\widetilde{Y}$ by [6, 9.2]. Thus $-I d_{E} \times I d_{E^{\prime}}$ gives rise to a biregular involution of the fiber product, $\widehat{W}$, which lifts to $\widetilde{W}$. There is a similar biregular involution of $\widetilde{W}$ coming from $I d_{E} \times\left(-I d_{E^{\prime}}\right)$. Taking the product gives a biregular action of $H$ on $\widetilde{W}$. This action commutes with the action of $G$ since it does on the generic fiber, $E \times_{K} E^{\prime} \times_{K} \widetilde{K}$.

Write $\bar{V}$ for the quotient of $\widetilde{W}$ by the action of $H \times G$. The proof of Proposition 2.3 yields

$$
H^{3}\left(\widetilde{W}, \mathcal{O}_{\widetilde{W}}\right)^{H \times G} \simeq H^{3}\left(\bar{V}, \mathcal{O}_{\bar{V}}\right) \simeq H^{3}\left(V, \mathcal{O}_{V}\right) .
$$

By Lemma $2.4, H^{3}\left(\widetilde{W}, \mathcal{O}_{\widetilde{W}}\right) \simeq H^{1}\left(\widetilde{X}, R^{1} \widetilde{\pi}_{*} \mathcal{O}_{\widetilde{Y}} \otimes R^{1} \widetilde{\pi}_{*}^{\prime} \mathcal{O}_{\widetilde{Y}^{\prime}}\right)$. Now $-I d \in H$ acts by multiplication by -1 on both $R^{1} \widetilde{\pi}_{*} \mathcal{O}_{\widetilde{Y}}$ and $R^{1} \widetilde{\pi}_{*}^{\prime} \mathcal{O}_{\widetilde{Y}}$ and hence acts trivially on $H^{3}\left(\widetilde{W}, \mathcal{O}_{\widetilde{W}}\right)$. Thus

$$
H^{3}\left(W, \mathcal{O}_{W}\right) \simeq H^{3}\left(\widetilde{W}, \mathcal{O}_{\widetilde{W}}\right)^{G} \simeq H^{3}\left(\widetilde{W}, \mathcal{O}_{\widetilde{W}}\right)^{H \times G} \simeq H^{3}\left(V, \mathcal{O}_{V}\right)
$$

(ii) This may be deduced from the triviality of the action of $H$ on $H^{3}(\mathcal{O})$ for an appropriate smooth, projective $k$-model of $E \times_{K} E^{\prime}$ over the composite field $\widetilde{K} K^{\prime}$ as in (i). Alternatively the assertion follows from Corollary 2.7 since the two expressions

$$
\frac{\operatorname{ord}_{x}\left(\Delta_{x}\right)+\operatorname{ord}_{x}\left(\Delta_{x}^{\prime}\right)}{12} \quad \text { and } \epsilon_{x}
$$


behave as follows under twisting by a quadratic extension branched at $x$ : For type $\mathrm{P}-\mathrm{P}^{*}$ or $\mathrm{I}_{n}-\mathrm{P}^{*}$ reduction both are unchanged; for type $\mathrm{I}_{n}-\mathrm{I}_{n^{\prime}}, \mathrm{P}-\mathrm{P}$, or $\mathrm{I}_{n}-\mathrm{P}$ reduction both increase by 1 ; for type $\mathrm{P}^{*}-\mathrm{P}^{*}$ both decrease by 1 . Indeed quadratic twisting changes $\operatorname{ord}_{x}\left(\Delta_{x}\right)$ by $6 \bmod 12$ (cf. [18, X.5.4(i)], the formula for $\Delta$ [18, p. 46 or p. 48] and [18, VII.1.3]) and leaves the order of the pole of the $J$-invariant, the $n$ in $\mathrm{I}_{n}$ and $\mathrm{I}_{n}^{*}$, unchanged. From Table 1 the effect on singular fibers is to interchange $\mathrm{I}_{n}$ and $\mathrm{I}_{n}^{*}, \mathrm{II}$ and $\mathrm{IV}^{*}$, III and III*, IV and $\mathrm{II}^{*}$. The asserted behaviour may now be read off Tables 1 and 2 .

\section{ACKNOWLEDGEMENT}

The author thanks the referee for comments and attention to detail.

\section{REFERENCES}

[1] Barth, W.; Peters, C.; Van de Ven, A. Compact complex surfaces. Ergebnisse der Mathematik und ihrer Grenzgebiete (3), 4. Springer-Verlag, Berlin, 1984. MR749574 (86c:32026)

[2] Chatzistamatiou, A.; Rülling, K., Higher direct images of the structure sheaf in positive characteristic, to appear in Algebra and Number Theory.

[3] Cossart, V.; Piltant, O., Resolution of singularities of threefolds in positive characteristic. II. J. Algebra 321 (2009), no. 7, 1836-1976. MR2494751 (2010c:14009)

[4] Cutkosky, S., Resolution of singularities for 3-folds in positive characteristic. Amer. J. Math 131 (2009), no. 1, 59-127. MR2488485 (2010e:14010)

[5] Donagi, R.; Ovrut, B.; Pantev, T.; Waldram, D., Standard-model bundles. Adv. Theor. Math. Phys. 5 (2001), no. 3, 563-615. MR1898371 (2003i:14012)

[6] Drinfeld, V., Elliptic modules, Math. USSR Sbornik 23 (1974), 561-592. MR0384707 (52:5580)

[7] Ellingsrud, G.; Lønsted, K., An equivariant Lefschetz formula for finite reductive groups, Math. Ann. 251 (1980), 253-261. MR589254 (81k:14039)

[8] Grothendieck, A., Sur quelques points d'algèbre homologique. Tôhoku Math. J. (2) 9 (1957), 119-121. MR0102537(21:1328)

[9] Hartshorne, R., Algebraic Geometry, Graduate Texts in Math. 52, Springer, New York, 1977. MR0463157(57:3116)

[10] Kollár, J., Lectures on Resolution of Singularities, Annals of Math. Studies 166, Princeton University Press, Princeton and Oxford, 2007. MR2289519 (2008f:14026)

[11] Liu, Q., Algebraic Geometry and Arithmetic Curves, Oxford Graduate Texts in Mathematics 6, Oxford University Press, Oxford, 2002. MR.1917232 (2003g:14001)

[12] Meyer, C., Modular Calabi-Yau Threefolds, Fields Institute Monograph 22, American Mathematical Society, Providence, RI, 2005. MR 2176545 (2007f:11070)

[13] Milne, J., Étale Cohomology, Princeton University Press, 1980. MR.559531 (81j:14002)

[14] Neukirch, J., Algebraic Number Theory, Grundlehren der Mathematischen Wissenschaften 322, Springer, 1999. MR1697859(2000m:11104)

[15] Saito, T., Vanishing cycles and geometry of curves over a discrete valuation ring. Amer. J. Math. 109 (1987), no. 6, 1043-1085. MR919003 (88h:14036)

[16] Schoen, C., Desingularized fiber products of semi-stable elliptic surfaces with vanishing third Betti number. Compos. Math. 145 (2009), no. 1, 89-111. MR2480496 (2010b:14077)

[17] Schoen, C.; Top, J., Drinfeld modules and torsion in the Chow groups of certain threefolds. Proc. Lond. Math. Soc. (3) 95 (2007), no. 3, 545-566. MR 2368276 (2008m:14020)

[18] Silverman, J., The Arithmetic of Elliptic Curves, Graduate Texts in Math. 106, Springer, 1986. MR817210 (87g:11070)

[19] Silverman, J., Advanced Topics in the Arithmetic of Elliptic Curves, Graduate Texts in Math. 151, Springer, New York, 1994. MR.1312368 (96b:11074)

[20] Viehweg, E., Rational singularities of higher dimensional schemes. Proc. A.M.S. 63 (1977), 6-8. MR0432637 (55:5624)

Department of Mathematics, Physics Bullding, Duke University, Research Drive, Durham, North Carolina 27708-0320 